\title{
Study on the Electrode Materials of Electrochemical Capacitor
}

\author{
Xiaolai Liu \& Jin Li (Corresponding author) \\ College of Science, Beijing University of Chemical Technology, Beijing 100029, China \\ E-mail: 11ltyx657@163.com
}

Received: December 20, 2010 Accepted: January 5, 2011 doi:10.5539/ijc.v3n2p198

\begin{abstract}
Super capacitor is a kind of new energy-storage component developed in recent years, with many advantages such as large energy-storage capacity, light quality, pollution free, multiple charging and discharging. Starting from the principle, development, and application of capacitor, the electrode materials including activated carbon fiber, carbon nano tube (CNT), ordered mesoporous carbon, and graphene used for the super capacitor are introduced in this article.
\end{abstract}

Keywords: Double-layer capacitor, Electrode materials, Graphene

\section{Introduction}

Electrochemical capacitor is a kind of new energy-storage component developed in recent years, with many advantages such as large energy-storage capacity, light quality, pollution free, multiple charging and discharging. Because of many advantages such as low cost, big specific surface area, good electrical conductivity, and simple preparation technique, the carbon has always been the first choice for the electrode materials of super capacitor. At present, the research and development of electrode materials are mainly concentrated in the carbon-based materials such as activated carbon, vitreous carbon, fiber, CNT, ordered mesoporous carbon, and graphene, and the mix with other materials. In this article, the development of super electrical capacitor, the research and development of relative new carbon-based materials are studied as follows.

\section{Introduction of electrochemical capacitor}

Electric energy is the most convenient and widely used energy in the world. It is mainly stored as two ways. First, it can be stored in the battery by the way of chemical energy, which needs the electrochemical activated matters produce the Faraday redox reaction to discharge electric charges. Second, it can be stored by the way of static electricity, i.e. it can be directly stored on the counter electrodes of capacitor by the form of negative charges and positive charges, which is the non-Faraday electric energy storage.

Except for large capacitors, general static capacitors only store a few electric charges, i.e. they only can store the electric energy by the lower energy density. However, at the interface of the charge electrode and solution, the double electrode layer exists (any two different phases will produce electric potential between them when they contact), induced by the electric charge separation. Two phases respectively have their own surplus charges with equal electric quantity, opposite electrodes, and these charges will attract each other, and form the double electrode layer, so the matter composed by the bet surface area will have the electrode with sufficient surface, and will produce large double-layer capacitor. When the double-layer capacitor discharges, the electrons only need to pass in and out on the electrode surface in the external circuit and the electrolytic zwitterions transfer from the interior of the solution to the interface of charge. Because of above causes, the charging and discharging process of capacitor is highly reversible.

\section{Carbon electrode materials of electrochemical capacitor}

\subsection{Activated carbon fiber}

Activated carbon fiber belongs to the high-efficient activated absorbing materials and the environmental protection engineering materials, with better property than the activated carbon. The preparation of the activated carbon fiber generally is to stabilize the organic precursor fiber in the low temperature (250 Celsius degree-450 Celsius degree). The organic fibers which are used for the activated carbon fiber precursor through carbonization and activation (600 Celsius degree-1100 Celsius degree) mainly include fibrin, polyacrylonitrile, pitch, phenolic aldehyde, and poval, and the application of activated carbon fiber is wider and wider in the double-layer capacitor. 
Zhang Yuqin et al dried phenolic aldehydes-base in the temperature of 80 Celsius degree for 12 hours, and heated up it to 600 Celsius degree by certain heating rate, and carbonized it for $60 \mathrm{~min}$, and dropped the temperature to the room temperature, and obtained the phenolic aldehyde carbon fiber. Mix the phenolic aldehydes-based carbon fiber with $\mathrm{KOH}$ according to the proportion of 1:4 and steep it in the water solution. Put it in the activation furnace after oven drying, and heat up to different temperatures (500 Celsius degree-900 Celsius degree) by certain heating rate and activate it for $60 \mathrm{~min}$, and cool it to the room temperature. Through neutralized processing, smash and screen after drying, obtain the electrode materials to prepare the double-layer capacitor.

\subsection{CNT}

From the electricity storage principle of capacitor, CNT is ideal electrode material. First, CNT is the cavity tube, with specific surface area, especially, single-wall CNT makes for forming the double electrode layer. In addition, the carbon forming the CNT belongs to SP hybridization which is linked by three hybrid bonds to form hexatomic ring, and in the hybridization, one hybrid bond is left to link the functional group to produce the Faraday reaction.

Liu, Chenguang et al used the organic matter catalytic pyrolysis to prepare the CNTs with the pipe diameter of $20 \mathrm{~nm}-40 \mathrm{~nm}$, and produced the wafer electrode on the nickel piece under the pressure of $6 \mathrm{MPa}$ through decomposing and decontaminating, used the electrolyte of $6 \mathrm{~mol} / \mathrm{LKOH}$ to test the charge and discharge by the current of 10MA, and the specific capacitance was $60 \mathrm{~F} / \mathrm{g}$.

Pico et al heatedly treated the single-wall CNT prepared by the electric arc method in the air in 300 Celsius degree-500 Celsius degree, prepared the electrode after adding the binder polyvinylidene chloride which mass fraction was $5 \%$, and tested the capacitance performance respectively by $6 \mathrm{~mol} / \mathrm{L} \mathrm{KON}$ and $2 \mathrm{~mol} / \mathrm{L}$ vitriol electrolyte. The specific capacitance of the single-wall CNT oxidized under 350 Celsius degree was $140 \mathrm{~F} / \mathrm{g}$, which was higher than the specific capacitance of the electrolyte composed by $2 \mathrm{~mol} / \mathrm{L}$ vitriol.

\subsection{Ordered mesoporous carbon}

Ordered mesoporous carbon is a kind of new carbon material, with big specific surface area and proper pore size distribution. There are three methods to prepare the ordered mesoporous carbon material, i.e. the catalysis and activation method, the mixed polymer carbonization method, and the moldboard method. Because the moldboard carbonization method can effectively control the pore size distribution, so its application foreground is more obvious. Since the researchers in Mobil Company in 1992 first synthetized the ordered mesoporous molecular sieve in M41S series, more molecular sieves with different structures have been synthetized one after another. The process can be approximately described as follow. First, "inject" appropriate carbon precursor matters into the pore cannel, and under certain catalysis condition, after carbonization and pyrogenic decomposition, remove the monox framework, and finally get the material of ordered mesoporous carbon.

Li Hongfang et al adopted the mesoporous monox molecular sieve SBA-15 as the moldboard, and the commercial hot-set phenolic resin as the carbon source (provided by Shanghai Qinan Glue Company, $\mathrm{M}=$ 5000-7000) to prepare the ordered mesoporous carbon. The synthesis of the SBA-15 moldboard material proceeded according to Zhao Dongyan's report. Put $1 \mathrm{~g}$ dry SBA-15 powder in the $10 \mathrm{~mL}$ phenolic resin ethanol solution with different $\mathrm{pH}$ indicators $(0.05-0.20 \mathrm{~g} \cdot \mathrm{mL}-1)$, and mix them in the normal temperature by the magnetic force until the ethanol solution volatilizes completely. Take the sample to the dryer with 100 Celsius degree and heat it for 2 hours, and solidify the phenolic resin, and then take it to the quartz tube furnace and heat it in nitrogen to 900 Celsius degree for 4 hours, and carbonize the phenolic resin completely. By removing silica in $10 \%$ of HF solution, the silica/carbon composite materials can produce a series of mesoporous carbon material, and then their electrochemical performances are tested.

\subsection{Graphene}

Graphene has special structure and excellent performance, and it is composed by a layer of dense carbon atoms packed on the honeycomb crystal lattice, and it is the thinnest two-dimensional material in the world, with the depth of $0.335 \mathrm{~nm}$. It can produce zero-dimensional fullerene, and crimp it to one-dimensional CNT, and accumulate to three-dimensional graphite. There are four synthesis methods of graphene. The first one is the micro-mechanical stripping method. It strips the slices of graphene from the surface of the graphite crystal by the mechanical force, and produces the flocculent flake crystals with single-layer graphene by using another kind of material to rub with pyrolytic graphite. The second one is the graphite intercalation method. Takes natural crystalline flake graphite as raw materials, and insert some non-carbon atoms, molecules, ions, or atom groups, and form the graphite intercalation composite. The third one is the epitaxy method. It uses the growth stromal 
atom structure to "grow" graphene, but the graphene produced by this method has not even depth, and the bond between graphene and base material will influence the characteristics of the carbon layer. The fourth one is the graphite-oxide deoxidation. After oxidation, the graphite will produce large numbers of hydrophilic functional groups, and through the adjustment of $\mathrm{pH}$ values of the solution under densely mechanical function, independently dispersive oxide graphite colloidal solution, which provides the possibility to prepare the graphene. Through chemical reduction, the stripped oxide graphite layer slices in the solution can be prepared as graphene.

D.S. Meryl et al suspended graphene oxide slices in the water, and deoxidated it by the hydrazine hydrate, and synthetized chemical modulated graphene (CMG). CMG is composed by the carbon slices with the depth of one atom, and it needs be functioned according to relative demands. The specific capacitances of the super capacitor which electrode materials are $\mathrm{CMG}$ are $135 \mathrm{~F} / \mathrm{g}$ and $99 \mathrm{~F} / \mathrm{g}$ in the $515 \mathrm{~mol} / \mathrm{L} \mathrm{KOH}$ and the organic electrolyte (1mol/ L tetraethyl four fluorine boric acid amines + ethylene or $1 \mathrm{~mol} / \mathrm{L}$ tetraethyl four fluorine boric acid amines + propylene chloride), and because of high conductivity of electrode materials, the capacitor has good performance in wide volt-ampere scanning speed. CMG has good conductivity and big surface area (basically be contacted completely), and it can be applied in capacitor, matching with general electrolyte system.

Yang Changling et al adopted improved Hummers method to prepare graphite oxide. Add 20g graphite powder, $10 \mathrm{~g}$ potassium peroxydisulfate, and $10 \mathrm{~g}$ phosphorus pentoxide in concentrated sulfuric acid with 80 Celsius degree, mix them evenly, and cool them for 6 hours, and wash them to the neutral solution, and dry it. Add dried sample in $230 \mathrm{~mL}$ concentrated sulfuric acid with 0 Celsius degree, and then add $60 \mathrm{~g}$ potassium permanganate, and keep the temperature of mixture below 20 Celsius degree, and bath it in the oil of 35 Celsius degree for 2 hours, and then slowly add $920 \mathrm{~mL}$ deionized water. After $15 \mathrm{~min}$, add deionized water, then the color of the mixture becomes bright yellow, and filter the mixture when it is still hot, and wash, filter, and dry it by the hydrochloric acid with $10 \%$ of concentration, get the graphite oxide. By test, the specific capacitance can achieve $138.6 \mathrm{~F} / \mathrm{g}$.

\section{Development prospect of electrochemical capacitor}

Super capacitor is a kind of new energy material between traditional capacitor and storage battery. Starting from the principle, development, and application of capacitor, the electrode materials including activated carbon fiber, carbon nano tube (CNT), ordered mesoporous carbon, and graphene used for the super capacitor are introduced in this article.

Though the carbon-based capacitor has been successfully commercialized, there are many problems to further enhance the performance of capacitor. To solve these problems, some new activated carbon materials, activation technologies, and adulteration of carbon materials still should be developed to essentially improve the performances of carbon-based materials.

\section{References}

Liu, Chenguang, Liu, Min \& Wang, Maozhang et al. (2002). Research and Development of Carbon Materials for Electrochemical Capacitors (Two): the Carbon Electrode. New Carbon Materials. No. 17(2). P.64.

Meryl D S, Park S J, Zhu Y W, et al. (2008). Graphene based ultracapacitors. Nano Lett. No. 8 (10). P. 3498-3502.

Pico F. Rojo J M. San Juan M L.et al. (2001). Single-walled carbon nanotubes as electrodes in supercapacitors. Advanced Materials. No.13(7). P.49.

Yang, Changling, Liu, Yunyun \& Sun, Yanping. (2010). Preparation of graphene and its electrochemical performance. Chinese Journal of Power Sources. No. 2. P.177-180. 